\title{
Variables De Afrontamiento Y Psicológicas Explicativas De La Ansiedad Escénica En Instrumentistas De Viento Metal
}

\author{
Francisco Javier Zarza-Alzugaray, Dr. \\ Oscar Casanova, Dr. \\ Santos Orejudo, Dr. \\ University of Zaragoza, Spain
}

doi: 10.19044/esj.2016.v12n34p24 URL:http://dx.doi.org/10.19044/esj.2016.v12n34p24

\begin{abstract}
In the process of musical learning, one of the biggest problems that students must face is stage anxiety. This problem can even lead to the abandonment of studies or lead students to adopt addictive behaviours related to the use of substances to mitigate the harmful consequences that stage anxiety can cause. In this study and taken into account the triple vulnerability system described by Barlow (2000) the presence of higher or lower levels of anxiety is explained from personal characteristics such as helplessness, sensitivity to punishment or dispositional optimism. Thus, it is also studied how these constructs are related to the existence of thoughts of abandonment of musical studies, as well as to the existence of behaviours related to the consumption of substances to mitigate the effects of stage anxiety. It becomes clear how helplessness is a key mediating factor between personal and contextual characteristics in order to generate higher or lower levels of stage anxiety. For this study has been gathered a sample of students of metal wind instruments from 6 different centres of Spanish higher education. In this sense we consider that more research is necessary, with other types of instrumentalists in order to study if there are differences between them that could suggest the application of specialized training plans in the future.
\end{abstract}

Keywords: Music education, music performance anxiety, optimism, sensitivity to punishment

\section{Resumen}

En el proceso de aprendizaje musical, uno de los mayores problemas a los que los estudiantes deben hacer frente es la ansiedad escénica. Este problema puede llegar incluso a propiciar el abandono de los estudios o 
llevar a los estudiantes a adoptar conductas adictivas relacionadas con el consumo de sustancias para mitigar las nefastas consecuencias que puede llegar a provocar la ansiedad escénica. En este estudio y a partir del triple sistema de vulnerabilidad descrito por Barlow (2000) se explica la presencia de mayores o menores niveles de ansiedad a partir de características personales como la indefensión, la sensibilidad al castigo o el optimismo disposicional. Se estudia también cómo estos constructos se relacionan con la existencia de pensamientos de abandono de los estudios musicales, así como con la existencia de conductas relacionadas con el consumo de sustancias para mitigar los efectos de la ansiedad escénica. Se hace patente cómo la indefensión es un factor mediador clave entre características personales y contextuales de cara a generar mayores o menores niveles de ansiedad escénica. Se ha contado con una muestra de estudiantes de instrumentos de viento metal de 6 centros distintos de formación superior españoles. Consideramos que es necesaria más investigación, con otros tipos de instrumentistas de cara a estudiar si existen diferencias entre ellos que sugieran la aplicación de planes formativos especializados en un futuro.

Palabras clave: educación musical, ansiedad escénica, optimismo, sensibilidad al castigo

\section{Introducción}

La ansiedad escénica en los estudiantes de música en etapas superiores (a todos los efectos estudios de nivel universitario) supone uno de los grandes problemas a los que tienen que hacer frente de manera recurrente en su etapa formativa (Papageorgi, Hallam y Welch, 2007). Como viene reflejado en los correspondientes currículos, una de las finalidades principales de la educación musical superior del instrumentista debe ser la de preparar a los estudiantes para las actuaciones públicas (Zarza, Casanova y Orejudo, 2016). Así, de cara a poder ofrecer recomendaciones y una mejor dimensión formativa tanto de docentes como de investigadores, se hace necesario contar con marcos teóricos que den cuenta y tengan capacidad explicativa de la presencia de ansiedad escénica y de las consecuencias que los niveles elevados de la misma pueden generar.

Salmon (1990) describió la ansiedad escénica en músicos como la experiencia de aprensión angustiosa que puede llegar a disminuir las habilidades interpretativas en un contexto público; hasta un grado que no garantiza la aptitud musical del individuo, el entrenamiento o el nivel de preparación de éste.

En lo referente a los modelos explicativos de la misma, vemos cómo Barlow (2000) indica que el origen de ésta, así como de otros problemas asociados con la ansiedad, son debidos a la interacción de tres factores de 
vulnerabilidad. El primer factor es el denominado factor de vulnerabilidad biológica y contempla rasgos de la personalidad como el afecto negativo, la inhibición del comportamiento o el neuroticismo. El segundo factor lo denomina factor de vulnerabilidad psicológica general y el tercer factor es el factor de vulnerabilidad específica. Según este modelo y acorde con Orejudo et al. (2016), los dos primeros factores de vulnerabilidad están asociados con las distintas características personales y contextuales; éstas terminan por propiciar perfiles de indefensión y control percibido mayores o menores de las situaciones habituales (Barlow, 2000; Gallagher, Bentley y Barlow, 2014). Así, la presencia de este mayor o menor control de la situación en contextos específicos como puedan ser las actuaciones musicales, nos llevan a la génesis de la antedicha vulnerabilidad psicológica específica, en donde se tienen en cuenta características personales y contextuales directamente relacionadas con la tarea a realizar.

Las principales repercusiones que podemos ver en las personas que sufren ansiedad escénica son dadas a nivel cognitivo, fisiológico y conductual. A nivel cognitivo encontramos pensamientos recurrentes negativos sobre la actuación que se va a realizar, problemas en el razonamiento o pérdidas de memoria. En lo fisiológico son típicos síntomas como las palmas de manos sudorosas, temblor de manos, piernas y voz, boca seca o incluso náuseas y vómitos en los casos más severos. Respecto al nivel conductual, encontramos conductas de afrontamiento como la evitación o escape, el consumo de sustancias para mitigar los efectos de la ansiedad, o incluso el abandono de las carreras musicales (Kenny, 2005; Papageorgi et al., 2007; Zarza, Casanova y Orejudo, 2016).

Por su parte, y relacionado con el constructo de la ansiedad, encontramos el optimismo disposicional; entendido éste como el rasgo de la personalidad en el que se contemplan expectativas positivas de cara a acontecimientos futuros, así como la confianza en que los objetivos marcados van a ser conseguidos. En este sentido mayores o menores niveles de optimismo disposicional han demostrado relación protectora de cara a generar problemas de ansiedad en la población (Hirsch et al., 2007; Mazé y Verlhiac, 2013).

Además, en esta investigación se maneja el constructo de la sensibilidad al castigo descrita por Gray (1982); en la que se explica la inhibición de la conducta en función de la sensibilidad personal que se tiene respecto a los posibles castigos en caso de que la realización de la tarea no sea la correcta o la esperada. En el caso de la sensibilidad al castigo, y tal y como describen Torrubia, Ávila, Moltó y Caseras (2001), subyace a la misma un factor ansioso de personalidad.

Teniendo en cuenta todo lo anterior, en este trabajo tratamos de encontrar un modelo explicativo que nos permita comprender de mejor modo 
cómo se explica la ansiedad escénica en estudiantes de cursos avanzados de música; asimismo, comprobar cómo influyen entre sí variables como el consumo de sustancias, los pensamientos de abandono, la indefensión, el optimismo disposicional o la sensibilidad al castigo en estos instrumentistas. Realizando, para alcanzar estos objetivos planteados, análisis estadísticos descriptivos y multivariados.

\section{Método}

\section{Participantes}

La muestra de este estudio estaba compuesta por 55 estudiantes (49 hombres y 6 mujeres) del Título Superior de Música pertenecientes a 6 centros de formación musical superior de España.

Estos estudiantes eran todos intérpretes de instrumentos de viento metal (10 trombón, 14 trompa, 22 trompeta, 6 tuba y 3 bombardino) y contaban con edades comprendidas entre los 17 y los 35 años $(=23.23$; D.T.=3.53).

\section{Procedimiento}

Los datos fueron recogidos directamente en las aulas de los estudiantes por distintos miembros del equipo de investigación y durante el mismo curso académico.

\section{Instrumentos y análisis de datos}

Para el mejor acopio de datos de los estudiantes se optó por la realización ad hoc de un instrumento específico que contenía la adaptación al español del Kenny Music Performance Anxiety Inventory de Kenny, Davis y Oates (2004) realizada por Zarza, Orejudo, Casanova y Mazas (2016). Esta versión presentó en su factor de ansiedad escénica un índice de fiabilidad medido con el $\alpha$ de Cronbach igual a .86 y está compuesta por 11 ítems. Del mismo modo la subescala de indefensión presentó un índice $\alpha$ de Cronbach igual a .78 con 10 ítems. Ambas subescalas están medidas con puntuación Likert de 7 puntos.

Respecto al optimismo disposicional se ha empleado la adaptación al castellano del LOT-R de Scheier, Carver y Bridges (1994) realizada por Otero et al. (1998). Es un instrumento de 10 ítems ( $\alpha$ de Cronbach igual a .78) en donde 3 de ellos evalúan optimismo, otros 3 ítems que deben ser invertidos evalúan pesimismo y otros 4 ítems son de relleno.

Relativo a la sensibilidad al castigo se ha empleado la subescala del cuestionario SPSRQ (Sensitivity to Punishment and Sensitivity to Reward Questionnaire) diseñado por Torrubia et al. (2001). Es una subescala dicotómica de 24 ítems que mide el Sistema de Inhibición de 
Comportamiento a partir de respuestas de Sí o No. En el proceso de validación presentó un $\alpha$ de Cronbach igual a .83.

En lo concerniente al tipo de análisis de los datos obtenidos a partir de los cuestionarios anteriormente indicados, decir que se realizaron empleando los programas estadísticos SPSS 19.0 y AMOS 2.0. Se llevaron a cabo ANOVA, análisis correlacionales $\mathrm{y}$ se ha planteado un modelo explicativo de la ansiedad escénica y el consumo de sustancias a partir de técnicas de análisis de ecuaciones estructurales.

\section{Resultados}

Un primer acercamiento a las distribuciones muestrales de las escalas indica que, a tenor de la no significatividad del estadístico K-S (p>.05), se puede asumir el principio de normalidad de todas ellas.

Respecto a la variable dependiente estudiada, la ansiedad escénica, vemos cómo ésta presenta una media (38.18; D.T.=12.47) significativamente menor ( $\mathrm{t}=-3.45 ; \mathrm{p}=.001)$ que la media teórica del instrumento que se sitúa en 44 puntos. Sin embargo, podemos ver también cómo para un porcentaje importante de los estudiantes, un 24\% de los mismos, presenta una media más elevada de la media teórica del instrumento y significativamente alejada ( $\mathrm{t}=-7.020 ; \mathrm{p}=.000)$, además, de la media de la muestra en más de una desviación típica.

En lo que concierne al consumo de sustancias vemos que el 34.5\% de los estudiantes ha consumido sustancias para mitigar los problemas de la ansiedad escénica mientras que un $65.5 \%$ no lo ha hecho. Respecto a los pensamientos de abandono vemos cómo un $16.4 \%$ sí que ha querido abandonar los estudios musicales debido a la ansiedad escénica mientras que un $83.6 \%$ no lo ha querido hacer nunca.

A nivel correlacional vemos cómo entre las características personales existen correlaciones significativas y directamente proporcionales entre la ansiedad escénica y la indefensión $(\mathrm{r}=.600 ; \mathrm{p}=.000)$ así como con la sensibilidad al castigo $(r=.628 ; \mathrm{p}=.000)$ mientras que es inversamente proporcional entre la indefensión y el optimismo disposicional $(r=.617$; $\mathrm{p}=.000$ ). Además, encontramos relación significativa y directamente proporcional entre el factor de indefensión y la sensibilidad al castigo $(\mathrm{r}=.585 ; \mathrm{p}=.000)$ mientras que es inversamente proporcional entre el optimismo y la indefensión $(\mathrm{r}=-.594 ; \mathrm{p}=.000)$ y la sensibilidad al castigo $(\mathrm{r}=-$ $.580 ; \mathrm{p}=.000)$. Tabla 1 .

Concerniente a los análisis de varianza en función de factores fijos como la querencia de abandono o el consumo de sustancias observamos cómo los niveles de ansiedad escénica son diferentes en función de los grupos poblacionales que se comparen. 
Tabla 1. Correlaciones entre variables psicológicas.

\begin{tabular}{ccccc}
\hline & $\begin{array}{c}\text { Ansiedad } \\
\text { escénica }\end{array}$ & Indefensión & Optimismo & $\begin{array}{c}\text { Sensibilidad al } \\
\text { castigo }\end{array}$ \\
\hline Ansiedad escénica & 1 & .600 & -.617 & .628 \\
Indefensión & & 1 & -.594 & .585 \\
Optimismo & & & 1 & -.580 \\
\hline
\end{tabular}

Vemos cómo los estudiantes que presentan conductas de consumo de sustancias afirmativas reportan una media (44.12; D.T.=11.10) significativamente mayor $\left(\mathrm{F}_{1,53}=7.345 ; \mathrm{p}=.009 ; \eta^{2}=.122\right)$ que los estudiantes que no han consumido sustancias (35.05; D.T. $=12.14)$ con un efecto del factor fijo moderado de en torno al $12.2 \%$ de la varianza del criterio de ansiedad escénica, acorde con lo indicado por Cohen (1988). Tabla 2.

Tabla 2. ANOVA. Variable Criterio: Ansiedad Escénica.

\begin{tabular}{ccccccc}
\hline Consumo & Media & Desv. típ. & N & F & Sig. & $\eta^{2}$ \\
\hline No & 35.05 & 12.14 & 36 & \multirow{2}{*}{7.345} & .009 & \multirow{2}{*}{.122} \\
Sí & 44.12 & 11.10 & 19 & & & \\
\hline
\end{tabular}

En lo que respecta a los valores de la ansiedad escénica y las posibles diferencias entre los estudiantes que hayan podido tener pensamientos de abandono o no abandono, advertimos cómo nuevamente existen diferencias de medias significativas $\left(\mathrm{F}=17.269 ; \mathrm{p}=.000 ; \eta^{2}=.246\right)$ entre el grupo de estudiantes que sí que han querido abandonar los estudios debido a la ansiedad escénica que padecen (52.04; D.T.=7.61) y los que no (35.47; D.T.=11.42); con un tamaño del efecto de los pensamientos de abandono sobre los niveles de ansiedad escénica elevado de en torno al $25 \%$ de la varianza del criterio de ansiedad, nuevamente acorde con Cohen (1988). Tabla 3.

Tabla 3. ANOVA. Variable Criterio: Ansiedad Escénica.

\begin{tabular}{ccccccc}
\hline Abandono & Media & Desv. típ. & $\mathrm{N}$ & $\mathrm{F}$ & Sig. & $\eta^{2}$ \\
\hline No & 35.47 & 11.42 & 46 & \multirow{2}{*}{17.269} & .000 & \multirow{2}{*}{.248} \\
Sí & 52.04 & 7.61 & 9 & & & \\
\hline
\end{tabular}

Llegados a este punto, podemos ver cómo de cara a explicar de mejor modo la ansiedad escénica se hace necesario el empleo de otras técnicas específicas como son los análisis a partir de modelos de ecuaciones estructurales. Así se ha planteado el modelo que puede verse en la Figura 1 en el cual la ansiedad escénica de manera significativa es explicada a partir de las características personales $\left(\chi_{6}^{2}=7.450 ; p=.281\right)$ en un aproximadamente $\mathrm{R}^{2}$ equivalente al 53.5\%. 


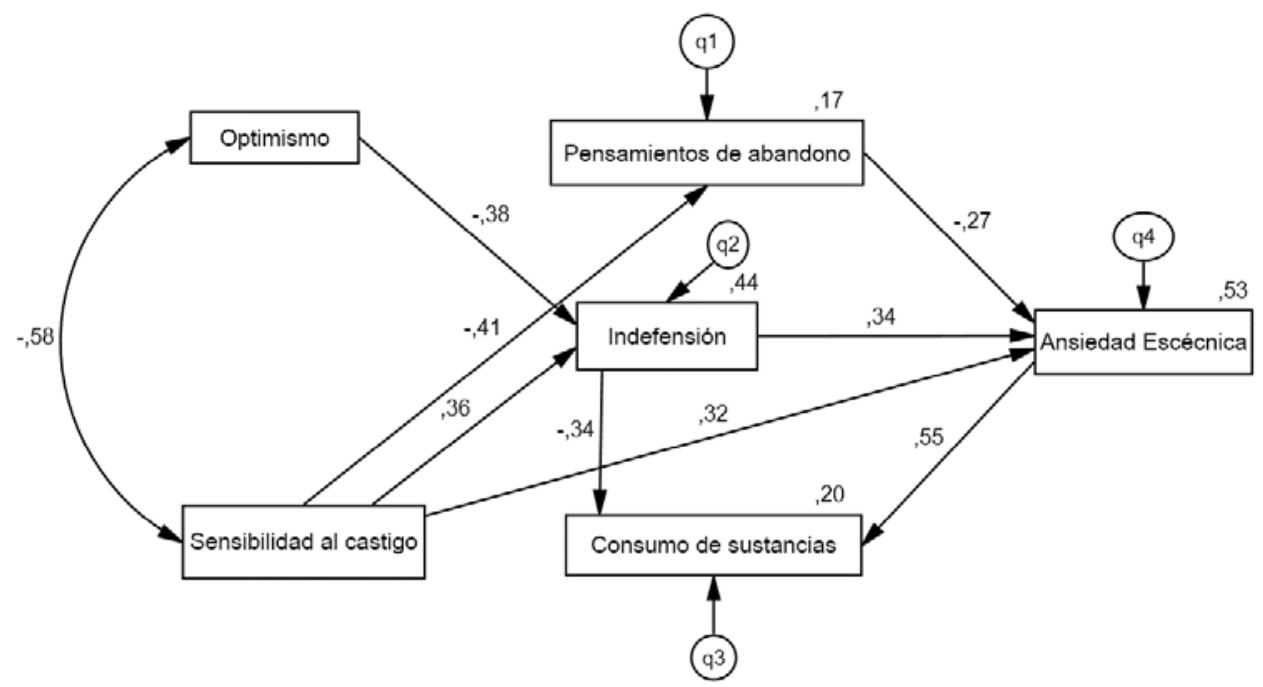

Figura 1. Modelo explicativo de la ansiedad escéncia y el consumo de sustancias.

Este modelo presenta unos datos de ajuste óptimos y encontramos que la relación CMIN/gl es igual a 1.242; p=.281; el índice CFI=.987; el RMSEA es igual a .067 (PCLOSE=.364). A su vez, los estimadores de la regresión presentan todos ello pesos significativos $(\mathrm{p}<.05)$. Tabla 4.

Tabla 4. Pesos de regresión estandarizados.

\begin{tabular}{cccc}
\hline Variable Criterio & Factor Fijo & $\beta$ & Sig. \\
\hline Indefensión & Castigo & .361 & .004 \\
Indefensión & Optimismo & -.385 & .002 \\
Abandono & Castigo & -.407 & .001 \\
Ansiedad Escénica & Abandono & -.272 & .007 \\
Ansiedad Escénica & Indefensión & .338 & .003 \\
Ansiedad Escénica & Castigo & .322 & .008 \\
Consumo & Indefensión & -.339 & .025 \\
Consumo & Ansiedad escénica & .550 & $* * *$ \\
\hline
\end{tabular}

De esta manera percibimos cómo de cara a explicar la ansiedad escénica, los pensamientos de abandono, las características personales de indefensión, así como la sensibilidad personal al castigo son factores que actúan de manera directa en la misma. Asimismo, vemos también el claro factor de mediación que tiene la indefensión entre el resto de factores fijos como el optimismo y la sensibilidad al castigo. También apreciamos cómo de cara a explicar las conductas de consumo de sustancias, el factor que presenta una aportación significativa mayor $(\beta=.550 ; \mathrm{p}=.000)$ es el factor específico de ansiedad. 


\section{Conclusión}

Se ha podido comprobar cómo la ansiedad escénica es un problema presente en esta muestra de estudiantes de música en etapa universitaria. Hay autores, como Orejudo et al. (2006), que indican que en estos periodos formativos superiores los problemas de ansiedad son habituales en la comunidad de estudiantes.

En porcentajes similares a los hallados por Zarza, Casanova y Robles (2016) y tal y como apunta Dalia (2004), se ha encontrado que en torno a $20 \%$ de los estudiantes reportan niveles medios de ansiedad mayores que la media. Por su parte, debemos hacer hincapié en que, tal y como se pone de manifiesto en otras investigaciones como la de Orejudo et al. (2016), nuevamente el factor de indefensión resulta un eje central en la explicación de la presencia de mayores o menores niveles de ansiedad; especialmente si atendemos a éste en su vertiente mediadora de otras características de la personalidad como el optimismo o la sensibilidad al castigo evaluada ésta a partir del sistema de inhibición de respuestas que, acorde con Gray (1982) y Torrubia et al. (2001), está ligado a la ansiedad. Del mismo modo también se ha podido constatar la importancia mediadora de la indefensión y del factor específico de ansiedad de cara a explicar las conductas relacionadas con el consumo de sustancias.

De acuerdo a Barlow (2000) estos resultados ponen de manifiesto la existencia previa y necesaria de un factor de vulnerabilidad psicológica general que relacionado con factores generales como el optimismo o la sensibilidad al castigo generen, junto con la acción de respuestas específicas como los pensamientos de abandono, un factor específico de vulnerabilidad; relacionado directamente en este caso con las actuaciones musicales públicas y que en la literatura científica denominamos ansiedad escénica.

Todas las conclusiones deben tomarse con las correspondientes precauciones debido al tamaño de la muestra disponible en este estudio. En esta ocasión se ha investigado a músicos estudiantes de unos instrumentos muy concretos, los de viento metal; sería necesario replicar el trabajo con otro tipo de familias instrumentales y compararlas entre sí. Las conclusiones obtenidas y las reflexiones provenidas de éstas darán una información muy valiosa respecto a un evidente problema que podrá derivar en propuestas concretas y soluciones formativas; creemos necesario que, en la formación de nuestros estudiantes de música, que en definitiva son los profesionales de la interpretación musical futura, se hace necesaria una mayor y mejor formación de cara a evitar o cuando menos aminorar las consecuencias que puede acarrear este problema. 


\section{References:}

1. Barlow, D. H. (2000). Unraveling the mysteries of anxiety and its disorders from the perspective of emotion theory. American Psychologist, 55(11), 1247-1263. doi:10.1037/0003-066X.55.11.1247

2. Cohen, J. (1988). Statistical Power Analysis for the Behavioral Sciences (2nd Ed.). Hillsdale, NJ: Erlbaum.

3. Dalia, G. (2004). Cómo superar la ansiedad escénica en músicos. Madrid: Mundimúsica.

4. Gallagher, M. W., Bentley, K. H. \& Barlow, D. H. (2014). Perceived control and vulnerability to anxiety disorders: A Meta-Analytic review. Cognitive Therapy Research, 38(6), 571-584. doi:10.1007/s10608-014-9624-X

5. Gray, J. A. (1982). Precis of the neuropsychology of anxiety: An enquiry into the functions of the septohippocampal system. The Behavioral and Brain Sciences, 5, 469-534.

6. Hirsch, J. K., Wolford, K., LaLonde, S. M., Brunk, L. \& Parker Morris, A. (2007). Dispositional optimism as a moderator of the relationship between negative life events and suicide ideation and attempts. Cognitive Therapy Research, 31, 533-546.

7. Kenny, D. T. (2005). A systematic review of treatments for music performance anxiety. Anxiety, Stress, and Coping, 18(3), 183-208.

8. Kenny, D. T., Davis, P. \& Oates, J. (2004). Music performance anxiety and occupational stress amongst opera chorus artists and their relationship with state and trait anxiety and perfectionism. Journal of Anxiety Disorders, 18(6), 757-777. doi:10.1016/j.janxdis.2003.09.004

9. Mazé, C. \& Verlhiac, J. F. (2013). Stress and coping strategies of first-year students: Distinctive roles of transactional and dispositional factors. Psychologie Francaise, 58, 89-105. doi:10.1016/j.psfr.2012.11.001

10. Orejudo, S., Nuño, J., Fernández, T., Ramos, M. T. \& Herrero, M. L. (2006). Participación del alumnado universitario en el aula. Una investigación sobre el temor a hablar en público en grandes grupos. Revista de Psicología General y Aplicada, 59, 257-276.

11. Orejudo, S., Zarza, F. J., Casanova, O., Rodríguez, C. \& Mazas, B. (2016). The relation of music performance anxiety (MPA) to optimism, self-efficacy, and sensitivity to reward and punishment: Testing Barlow's theory of personal vulnerability on a sample of Spanish music students. Psychology of Music, November 11, 1-14. doi:10.1177/0305735616674791

12. Otero, J. M., Luengo, A., Romero, E. Gómez, J. A. \& Castro, C. (1998). Psicología de personalidad. Manual de prácticas. Barcelona: Ariel Practicum. 
13. Papageorgi, I., Hallam, S. \& Welch, G. (2007). A conceptual framework for understanding musical performance anxiety. Research Studies in Music Education, 28, 83-107. doi:10.1177/1321103X070280010207

14. Salmon, P. (1990). A psychological perspective on Musical Performance Anxiety: A review of the literature. Medical Problems of Performing Artists, 5(1), 1-11.

15. Scheier, M. F., Carver, C. S. \& Bridges, M. W. (1994). Distinguishing optimism from neuroticism (and trait anxiety, self-mastery, and selfesteem): A reevaluation of the Life Orientation Test. Journal of Personality and Social Psychology, 51, 1257-1264.

16. Torrubia, R., Ávila, C., Moltó, J. \& Caseras, X. (2001). The sensitivity to punishment and sensitivity to reward questionnaire (SPSRQ) as a measure of Gray's anxiety and impulsivity dimensions. Personality and Individual Differences, 31(6), 837-862. doi:10.1016/S0191-8869(00)00183-5

17. Zarza, F. J., Casanova, O. \& Orejudo, S. (2016). Ansiedad escénica y constructos psicológicos relacionados. Estudiantes de cinco conservatorios superiores de música españoles. RIEM. Revista Internacional de Educación Musical, 4, 13-24. doi:10.12967/RIEM2016-4-p013-024

18. Zarza， F. J., Casanova, O. \& Robles, J. (2016). Relación entre ansiedad escénica, perfeccionismo y calificaciones en estudiantes del Título Superior de Música. ReiDoCrea. Revista electrónica de investigación y Docencia Creativa, 5, 16-21.

19. Zarza, F. J., Orejudo, S., Casanova, O. \& Mazas, B. (2016). Kenny Music Performance Anxiety Inventory: Confirmatory factor analysis of the Spanish version. Psychology of Music, 44(3), 340-352. doi:10.1177/0305735614567932 